\title{
Pulling an adsorbed polymer chain off a solid surface
}

\author{
S. Bhattacharya ${ }^{1, a}$, A. Milchev ${ }^{1,2}$, V.G. Rostiashvili ${ }^{1}$, and T.A. Vilgis ${ }^{1}$ \\ 1 Max Planck Institute for Polymer Research, 10 Ackermannweg, 55128 Mainz, Germany \\ 2 Institute for Physical Chemistry, Bulgarian Academy of Science, 1113 Sofia, Bulgaria
}

Received 1 April 2009 and Received in final form 29 May 2009

Published online: 16 July 2009 - (c) EDP Sciences / Società Italiana di Fisica / Springer-Verlag 2009

\begin{abstract}
The thermally assisted detachment of a self-avoiding polymer chain from an adhesive surface by an external force applied to one of the chain-ends is investigated. We perform our study in the "fixed height" statistical ensemble where one measures the fluctuating force, exerted by the chain on the last monomer when a chain-end is kept fixed at height $h$ over the solid plane at different adsorption strength $\epsilon$. The phase diagram in the $h-\epsilon$ plane is calculated both analytically and by Monte Carlo simulations. We demonstrate that in the vicinity of the polymer desorption transition a number of properties like fluctuations and probability distribution of various quantities behave differently, if $h$ rather than $f$ is used as an independent control parameter.
\end{abstract}

PACS. 82.35.Gh Polymers on surfaces; adhesion - 64.60.A- Specific approaches applied to studies of phase transitions $-62.25 .+\mathrm{g}$ Mechanical properties of nanoscale systems

\section{Introduction}

The properties of single polymer chains at surfaces have received considerable attention in recent years. Much of this has been spurred by new experimental techniques such as atomic force microscopy (AFM) and optical/magnetic tweezers [1] which allow one to manipulate single polymer chains. Study of single polymer molecules at surfaces, such as mica or self-assembled monolayers, by Atomic Force Microscopy (AFM) method provides a great scope for experimentation [2-8]. Applications range from sequential unfolding of collapsed biopolymers over stretching of coiled synthetic polymers to breaking individual covalent bonds [9-11].

In these experiments it is customary to anchor a polymer molecule with one end to the substrate whereas the other end is fixed on the AFM cantilever. The polymer molecule can be adsorbed on the substrate while the cantilever recedes from the substrate. In so doing one can prescribe the acting force in AFM experiment whereas the distance between the tip and the surface is measured. Conversely, it is also possible to fix the distance and measure the corresponding force, a method which is actually more typical in AFM experiments. From the standpoint of statistical mechanics these two cases could be qualified as $f$-ensemble (the force is fixed while the fluctuating chainend height is measured) and as $h$-ensemble ( $h$ is fixed while one measures the fluctuating $f$ ). Recently these two ways of descriptions as well as their interrelation were discussed for the case of a phantom polymer chain by Skvortsov et

\footnotetext{
a e-mail: bhattach@mpip-mainz.mpq.de
}

al. [12]. In recent papers $[13,14]$ we studied extensively a single-tethered self-avoiding polymer adsorption on a solid substrate with an external force applied to a free chain's end in the $f$-ensemble.

In the present paper we consider the detachment process of a single self-avoiding polymer chain, keeping the distance $h$ between the free chain's end and the substrate as the control parameter. We derive analytical results for the main observables which characterize the detachment process. The mean value as well as the probability distribution function (PDF) of the order parameter are presented in close analytical expressions using the Grand Canonical Ensemble (GCE) method [14]. The basic forceheight relationship which describes the process of polymer detachments by pulling, that is, the relevant equation of state for this system is also derived both for extendible as well as for rigid bonds and shown to comply well with our Monte Carlo simulation results. We demonstrate also that a number of properties behave differently in the vicinity of the phase transition, regarding which of the two equivalent ensembles is used as a basis for the study of systems's behavior.

\section{Single-chain adsorption: Using distance as a control parameter}

\subsection{Deformation of a tethered chain}

Before considering the adsorption-desorption behavior of a polymer in terms of the chain-end distance $h$, we first 
examine how a chain tethered to a solid surface responds to stretching. This problem amounts to finding the chain free end probability distribution function (PDF) $P_{N}(h)$, where $N$ is the chain length, i.e., the number of beads. The partition function of such a chain at fixed distance $h$ of the chain-end from the anchoring plane is given by

$$
\Xi_{\text {tail }}(N, h)=\frac{\mu_{3}^{N}}{N^{\beta}} l_{0} P_{N}(h),
$$

where $\beta=1-\gamma_{1}$ and the exponent $\gamma_{1}=0.680$ [15]. Here $\mu_{3}$ is a model-dependent connective constant (see, e.g. ref. [15]). In eq. (1) $l_{0}$ denotes a short-range characteristic length which depends on the chain model. Below we discuss the chain deformation within two models: the bead-spring (BS) model for elastic bonds and the freely jointed bond vectors (FJBV) model where the bonds between adjacent beads are considered rigid.

\subsubsection{Bead-spring model}

The form of $P_{N}(h)$ has been discussed earlier [16,17] and later used in studies of the monomer density in polymer brushes [18]. Here we outline this in a way which is appropriate for our purposes. The average end-to-end chain distance reads $R_{N}=l_{0} N^{\nu}$, where $l_{0}$ is the mean distance between two successive beads on a chain and $\nu \approx 0.588$ is the Flory exponent [19]. The short-distance behavior, $h \ll R_{N}$, is given by

$$
P_{N}(h) \propto\left(\frac{h}{R_{N}}\right)^{\zeta}
$$

where the exponent $\zeta \approx 0.8$. For the long-distance behavior, $h / R_{N} \gg 1$, we assume, following ref. [18], that the PDF of the end-to-end vector $\mathbf{r}$ is given by des Cloizeaux's expression [20] for a chain in the bulk: $P_{N}(\mathbf{r})=\left(1 / R_{N}\right) F\left(\mathbf{r} / R_{N}\right)$, where the scaling function $F(x) \propto x^{t} \exp \left[-D x^{\delta}\right]$, and the exponents $t=(\beta-d / 2+$ $\nu d) /(1-\nu), \delta=1 /(1-\nu)$. Here and below $d$ denotes the space dimensionality. One should emphasize that the presence of a surface is manifested only by the replacement of the universal exponent $\gamma$ with another universal exponent $\gamma_{1}$ (as compared to the pure bulk case!). By integration of $P_{N}(\mathbf{r})$ over the $x$ and $y$ coordinates while $h$ is measured along the $z$-coordinate, one obtains $P_{N}(h) \propto\left(h / R_{N}\right)^{2+t-\delta} \exp \left[-D\left(h / R_{N}\right)^{\delta}\right]$. As the longdistance behavior is dominated mainly by the exponential function while the short-distance regime is described by eq. (2), we can approximate the overall behavior as

$$
P_{N}(h)=\frac{A}{R_{N}}\left(\frac{h}{R_{N}}\right)^{\zeta} \exp \left[-D\left(\frac{h}{R_{N}}\right)^{\delta}\right] .
$$

A comparison of the distribution, eq. (3), with our simulation data is shown in fig. 1 . The constants $A$ and $D$ in eq. (3) can be found from the conditions: $\int P_{N}(h) \mathrm{d} h=1$ and $\int h^{2} P_{N}(h) \mathrm{d} h=R_{N}^{2}$. This leads to

$$
A=\delta\left[\Gamma\left(\frac{1+\zeta}{\delta}\right)\right]^{-(1+\zeta) / 2}\left[\Gamma\left(\frac{3+\zeta}{\delta}\right)\right]^{-(1-\zeta) / 2},
$$

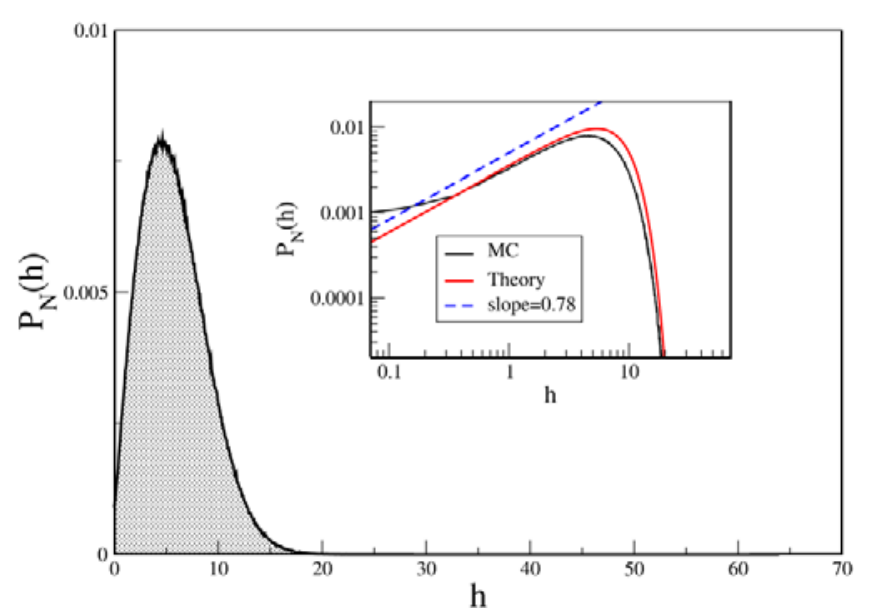

Fig. 1. Probability distribution $P_{N}(h)$ of chain-end positions $h$ above the grafting plane for a polymer with $N=128$ monomers at zero strength of the adsorption potential $\epsilon=0.0$. In the inset the MC data for $P_{N}(h)$ (solid black line) is compared to the theoretic result, eq. (3). Dashed line denotes the expected slope of $\zeta \approx 0.78$ of the probability distribution for small heights.

and

$$
D=\left[\Gamma\left(\frac{3+\zeta}{\delta}\right)\right]^{\delta / 2}\left[\Gamma\left(\frac{1+\zeta}{\delta}\right)\right]^{-\delta / 2}
$$

where $\delta \approx 2.43$ and $\zeta \approx 0.8$. One gets thus the estimates $A \approx 2.029$ and $D \approx 0.670$.

The free energy of the tethered chain with a fixed distance $h$ takes on the form $F_{\text {tail }}(N, h)=-k_{B} T \ln \Xi_{\text {tail }}$ $(N, h)$, where $k_{B}$ denotes the Boltzmann constant. By making use of eqs. (1) and (3), the expression for the force $f_{N}$, acting on the end monomer when kept at distance $h$ is given by

$f_{N}=\frac{\partial}{\partial h} F_{\text {tail }}(N, h)=\frac{k_{B} T}{R_{N}}\left[\delta D\left(\frac{h}{R_{N}}\right)^{\delta-1}-\zeta\left(\frac{R_{N}}{h}\right)\right]$.

One should note that at $h / R_{N} \gg 1$ we have $h \propto$ $R_{N}\left(R_{N} f_{N} / k_{B} T\right)^{1 /(\delta-1)}$ which, after taking into account that $\delta^{-1}=1-\nu$, leads to the well-known Pincus deformation law: $h \propto l_{0} N\left(l_{0} f_{N} / k_{B} T\right)^{1 / \nu-1}$ [21]. Within the framework of this approximation the (dimensionless) elastic energy reads $U_{\mathrm{el}} / k_{B} T=-N\left(l_{0} f_{N} / k_{B} T\right)^{1 / \nu}$. In result the corresponding free energy of the chain tail is given by

$$
\frac{F_{\text {tail }}}{k_{B} T}=-N\left(\frac{l_{0} f_{N}}{k_{B} T}\right)^{1 / \nu}-N \ln \mu_{3} .
$$

Equation (6) indicates that there exists a height $h_{0}=$ $(\zeta / \delta D)^{1 / \delta} R_{N}$ over the surface where the force $f_{N}$ changes sign and becomes negative (that is, the surface repulsion dominates). According to eq. (6) the force diverges as $f_{N} \propto-k_{B} T / h$ upon further decrease of the distance $h$.

\subsubsection{Freely jointed chain}

It is well known [19] that the Pincus law, eq. (6), describes the deformation of a linear chain at intermediate 
force strength, $1 / N^{\nu} \ll l_{0} f_{N} / k_{B} T \leq 1$. Direct Monte Carlo simulation results indicate that, depending on the model, deviations from Pincus law emerge at $h / R_{N} \geq 3$ (bead-spring off-lattice model) [22], or $h / R_{N} \geq 6$ (Bond Fluctuation Model) [23]. In such "overstretched" regime (when the chain is stretched close to its contour length) one should take into account that the chain bonds cannot expand indefinitely. This case could be treated within the simple freely jointed bond vectors (FJBV) model $[22,24]$ where the bond length $l_{0}$ is fixed. In this model the forcedeformation relationship is given by

$$
f_{N}=\frac{k_{B} T}{l_{0}} \mathcal{L}^{-1}\left(\frac{h}{l_{0} N}\right),
$$

where $\mathcal{L}^{-1}$ denotes the inverse Langevin function $\mathcal{L}(x)=$ $\operatorname{coth}(x)-1 / x$ and $l_{0}$ is the fixed bond vector length. We discuss the main results pertaining to the FJBV model in appendix A. The elastic deformation energy reads $U_{\mathrm{el}} / k_{B} T=-\left(l_{0} f_{N} / k_{B} T\right) \sum_{i=1}^{N}\left\langle\cos \theta_{i}\right\rangle=$ $-N\left(l_{0} f_{N} / k_{B} T\right) \mathcal{L}\left(l_{0} f_{N} / k_{B} T\right)$, where $\theta_{i}$ is the average polar angle of the $i$-th bond vector (see appendix A). Thus the corresponding free energy of the chain tail for the FJBV model reads

$$
\frac{F_{\text {tail }}}{k_{B} T}=-N \mathcal{G}\left(\frac{l_{0} f_{N}}{k_{B} T}\right)-N \ln \mu_{3},
$$

where we have used the notation $\mathcal{G}(x)=x \mathcal{L}(x)=$ $x \operatorname{coth}(x)-1$. Now we are in a position to discuss the pulling of the adsorbed chain controlled by the chain height $h$.

\subsection{Pulling controlled by the chain-end position}

Consider now an adsorbed chain when the adsorption energy per monomer is sufficiently large, $\varepsilon \geq \varepsilon_{c}$, where $\varepsilon_{c}$ denotes a corresponding critical energy of adsorption. Below we will also use the notation $\epsilon=\varepsilon / k_{B} T$ for the dimensionless adsorption energy. The problem of force-induced polymer desorption could be posed as follows: how is the process of polymer detachment governed by the chain-end position $h$ ? Figure 2a gives a schematic representation of such a system, and the situation in a computer experiment, as shown in the snapshot fig. $2 \mathrm{~b}$, is very similar.

As is evident from fig. $2 \mathrm{a}$, the system is built up from a tail of length $M$ and an adsorbed portion of length $N-M$. The adsorbed part can be treated within the GCE approach [14]. In our earlier treatment, ref. [14], it was shown that the free energy of the adsorbed portion is $F_{\text {ads }}=k_{B} T(N-M) \ln z^{*}(\epsilon)$, where the fugacity per adsorbed monomer $z^{*}(\epsilon)$ depends on $\epsilon$ and can be found from the basic equation

$$
\Phi\left(\alpha, \mu_{3} z^{*}\right) \Phi\left(\lambda, \mu_{2} w z^{*}\right)=1 .
$$

The so-called polylog function in eq. (10) is defined as $\Phi(\alpha, z)=\sum_{n=1}^{\infty} z^{n} / n^{\alpha}$ and the connective constants $\mu_{3}$, $\mu_{2}$ in three- and two-dimensional space have values which
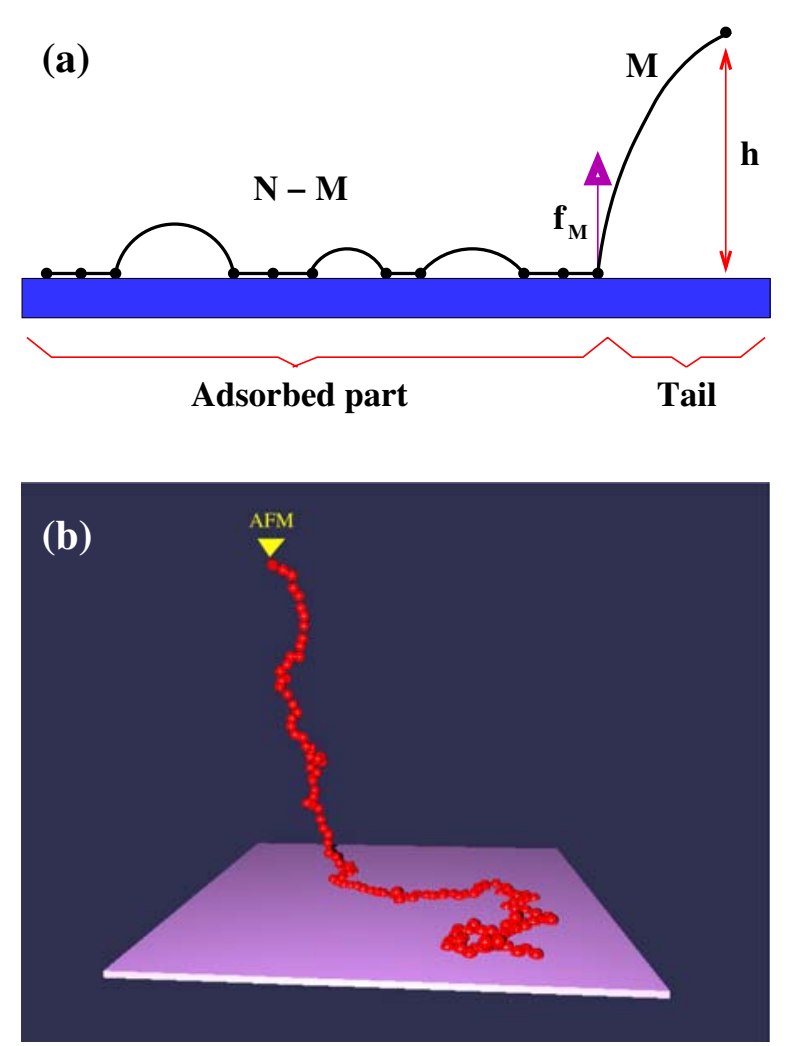

Fig. 2. (a) Schematic graph of an adsorbed polymer chain, partially detached from the plane by an external force which keeps the last monomer at height $h$. The total chain is built up from a tail of length $M$ and an adsorbed part of length $N-M$. The force $f_{M}$ acting on the chain-end is conjugated to $h$, i.e., $f_{M}=\partial F_{\text {tail }} / \partial h$. (b) A snapshot from the MC simulation: $N=$ $128, h=25.0, \epsilon=4.0$ and $\langle f\rangle=6.126$.

are model dependent [15]. The exponents $\alpha=1+\phi$ and $\lambda=1-\gamma_{d=2}$ where $\phi \approx 0.5$ is the crossover exponent which governs the polymer adsorption at criticality, and in particular, the fraction of adsorbed monomers at the critical adsorption point (CAP) $\epsilon=\epsilon_{c}$. The constant $\gamma_{d=2}=1.343$ [15]. Finally $w=\exp (\epsilon)$ is the additional statistical weight gained by each adsorbed segment.

In equilibrium, the force conjugated to $h$, that is, $f_{M}=$ $\partial F_{\text {tail }} / \partial h$, should be equal to the chain resistance force to pulling $f_{\mathrm{p}}=\left(k_{B} T / l_{0}\right) \mathcal{F}(\epsilon)$ (where $\mathcal{F}(\epsilon)$ is a scaling function depending only on $\epsilon)$, i.e.,

$$
\begin{aligned}
& f_{M}= \\
& \left\{\begin{array}{l}
\frac{k_{B} T}{R_{M}}\left[\delta D\left(\frac{h}{R_{M}}\right)^{\delta-1}-\zeta\left(\frac{R_{M}}{h}\right)\right]=f_{\mathrm{p}}, \quad \text { for BS model, } \\
\frac{k_{B} T}{l_{0}} \mathcal{L}^{-1}\left(\frac{h}{l_{0} M}\right)=f_{\mathrm{p}}, \quad \text { for FJBV model. }
\end{array}\right.
\end{aligned}
$$

The resisting force $f_{\mathrm{p}}$ holds the last adsorbed monomer on the adhesive plane (see again fig. 2a whereby this monomer experiences a force $f_{M}$ ). One should emphasize that the force $f_{\mathrm{p}}$ stays constant in the course of the pulling process as long as one monomer, at least, is adsorbed on the surface. Thus $f_{\mathrm{p}}$ corresponds to a plateau 
on the deformation curve (force $f$ vs. chain-end position $h$ ). The adsorbed monomer (see fig. 2) has a chemical potential, $\mu_{\text {ads }}=\ln z^{*}$, which in equilibrium should be equal to the chemical potential of a desorbed monomer in the tail, $\mu_{\text {des }}=\partial\left(F_{\text {tail }} / k_{B} T\right) / \partial N$. The expression for $F_{\text {tail }}$ depends on the model and is given either by eq. (7) for the BS model or by eq. (9) in the case of FJBV model. Taking this into account, the condition $\mu_{\mathrm{ads}}=\mu_{\mathrm{des}}$ leads to the following "plateau law" relationship

$$
\frac{l_{0} f_{\mathrm{p}}}{k_{B} T}=\left\{\begin{array}{l}
\left|\ln \left[\mu_{3} z^{*}(\epsilon)\right]\right|^{\nu}, \quad \text { for BS model, } \\
\mathcal{G}^{-1}\left(\left|\ln \left[\mu_{3} z^{*}(\varepsilon)\right]\right|\right), \quad \text { for FJVB model, }
\end{array}\right.
$$

where $\mathcal{G}^{-1}(x)$ stands for the inversion of the function $\mathcal{G}(x)=x \operatorname{coth}(x)-1$. One should note that eq. (12) coincides with eq. (3.16) in ref. [14] which determines the detachment line in the pulling controlled by the applied force. Close to the critical point $\epsilon_{c}$, the plateau force $f_{\mathrm{p}}$ goes to zero. Indeed, since in the vicinity of the critical point $\ln \left[\mu_{3} z^{*}(\epsilon)\right] \propto-\left(\epsilon-\epsilon_{c}\right)^{1 / \phi}$ (see ref. [14]), and $\mathcal{G}^{-1}(x) \approx(3 x)^{1 / 2}$, one may conclude that $f_{\mathrm{p}} \propto\left(\epsilon-\epsilon_{c}\right)^{\nu / \phi}$ for the BS model and $f_{\mathrm{p}} \propto\left(\epsilon-\epsilon_{c}\right)^{1 / 2 \phi}$ for the FJVB model.

One can resolve eq. (11) with respect to $M$ (taking into account that $h \gg R_{M}$ ), and arrive at the expression for the tail length

$$
M(h, \epsilon)= \begin{cases}\frac{h}{l_{0}}\left(\frac{k_{B} T}{l_{0} f_{\mathrm{p}}}\right)^{1 / \nu-1}, & \text { for BS model } \\ \frac{h}{l_{0}}\left[\mathcal{L}\left(\frac{l_{0} f_{\mathrm{p}}}{k_{B} T}\right)\right]^{-1}, & \text { for FJVB model },\end{cases}
$$

where the force at the plateau, $f_{\mathrm{p}}$, is described by eq. (12). If for the degree of adsorption one uses as an order parameter the fraction of chain contacts with the plane, $n=N_{s} / N$, where $N_{s}$ is the number of monomers on the surface, one can write [14]

$$
n=-\frac{1}{k_{B} T N} \frac{\partial}{\partial \epsilon}\left(F_{\mathrm{ads}}+F_{\text {tail }}\right)
$$

where $F_{\text {ads }}$ and $F_{\text {tail }}$ are free energies of the adsorbed and desorbed portions of the chain, respectively. The free energy $F_{\text {ads }}=k_{B} T[N-M(h, \epsilon)] \ln z^{*}(\epsilon)$, whereas $F_{\text {tail }}=k_{B} T \mu_{\text {des }} M(h, \epsilon)$ (recall that $\mu_{\text {des }}$ is the chemical potential of a desorbed monomer). After substitution of these expressions in eq. (14) and taking into account that in equilibrium $\mu_{\mathrm{ads}}=\mu_{\mathrm{des}}$ (the sequence of operations is important: the derivation with respect to $\epsilon$ to be followed by the condition $\left.\mu_{\mathrm{ads}}=\mu_{\mathrm{des}}\right)$, one gets

$$
n=-\left[1-\frac{M(h, \epsilon)}{N}\right] \frac{\partial \ln z^{*}(\epsilon)}{\partial \epsilon},
$$

i.e. the order parameter $n$ is defined by the product of monomer fraction in the adsorbed portion, $1-M / N$, and the fraction of surface contacts in this portion, $-\partial \ln z^{*} / \partial \epsilon$. The expressions for the order parameter can be recast in the form

$$
\begin{aligned}
& n=\left|\frac{\partial \ln z^{*}(\epsilon)}{\partial \epsilon}\right| \\
& \times \begin{cases}1-\frac{h}{c_{1} l_{0} N}\left(\frac{k_{B} T}{l_{0} f_{\mathrm{p}}}\right)^{1 / \nu-1}, & \text { for BS model, } \\
1-\frac{h}{c_{2} l_{0} N}\left[\mathcal{L}\left(\frac{l_{0} f_{\mathrm{p}}}{k_{B} T}\right)\right]^{-1}, & \text { for FJVB model. }\end{cases}
\end{aligned}
$$

Here $c_{1}$ and $c_{2}$ are some constants of the order of unity.

As one can see from eq. (16), the order parameter decreases linearly and steadily with $h / N$. This behavior is qualitatively different from the abrupt jump of $n$ when the pulling force $f$ is changed as a control parameter. In sect. 5 we will show that this predictions is in a good agreement with our MC findings. The transition point on the $n v s . h$ curve corresponds to total detachment, $n=0$. The corresponding distance $h$ will be termed a "detachment height" $h_{D}$. The dependence of $h_{D}$ on the adsorption energy $\epsilon$ can be obtained from eq. (16) where $n$ is set to zero, i.e.

$$
\frac{h_{D}}{l_{0} N}= \begin{cases}\left(\frac{l_{0} f_{\mathrm{p}}}{k_{B} T}\right)^{1 / \nu-1}, & \text { BS model } \\ \mathcal{L}\left(\frac{l_{0} f_{\mathrm{p}}}{k_{B} T}\right), & \text { FJVB model }\end{cases}
$$

where again $f_{\mathrm{p}}$ as a function of $\epsilon$ is given by eq. (12). The line given by eq. (17), is named "detachment line". It corresponds to an adsorption - desorption polymer transition which appears as of second order since this order parameter $n$ goes to zero continuously as $h$ increases. One should emphasize, however, that this "detachment" transition has the same nature as the force-induced desorption transition [14] in the $f$-ensemble where the pulling force $f$, rather than the distance $h$, is fixed and used as a control parameter. This phase transformation is known to be of first order.

It is easy to understand (cf. with eq. (13)) that the condition $M(h, \varepsilon)=N$ corresponds to the detachment line as well as to a terminal point of the force plateau. It can be seen in the MC - simulation results in sect. 5 , fig. 8 .

\section{Probability distribution $P(K)$ of the number of adsorbed monomers}

The grand canonical ensemble (GCE) method, which has been used in our recent paper [14], is a good starting point to calculate the probability distribution function $P(K)$ of the adsorbed monomers number $K$. According to this approach, the GCE partition function of an adsorbed chain has the form

$$
\Xi(z, w)=\sum_{N=1}^{\infty} \sum_{K=0}^{\infty} \Xi_{N, K} z^{N} w^{K}=\frac{V_{0}(w z) Q(z)}{1-V(w z) U(z)},
$$

where $z$ and $w=\exp (\epsilon)$ are the fugacities conjugated to chain length $N$ and to the number of adsorbed monomers $K$, respectively. In eq. (18) $U(z), V(w z)$ and $Q(z)$ denote 
the GCE partition functions for loops, trains and tails, respectively. The building block adjacent to the tethered chain end corresponds to $V_{0}(w z)=1+V(w z)$. It has been shown [14] that the functions $U(z), V(w z)$ and $Q(z)$ can be expressed in terms of polylog functions, defined in the paragraph after eq. (10), as $U(z)=\Phi\left(\alpha, \mu_{3} z\right), V(w z)=$ $\Phi\left(1-\gamma_{d=2}, \mu_{2} w z\right)$ and $Q(z)=1+\Phi\left(1-\gamma_{1}, \mu_{3} z\right)$, where $\mu_{3}$ and $\mu_{2}$ are $3 d$ and $2 d$ connective constants respectively. By making use of the inverse Laplace transformation of $\Xi(z, w)$ with respect to $z$ (see, e.g., [25]) the partition function (canonical with respect to the chain length $N$ ) is obtained as

$$
\Xi_{N}(w)=\sum_{K=0}^{\infty} \Xi_{N, K} w^{K}=\exp \left[-N \ln z^{*}(w)\right],
$$

where $z^{*}(w)$ is a simple pole of $\Xi(z, w)$ in the complex $z$ plane given by equation $V\left(w z^{*}\right) U\left(z^{*}\right)=1$, i.e. by eq. (10).

The (non-normalized) probability for the chain to have $K$ adsorbed monomers is $P(K) \propto \Xi_{N, K} \mathrm{e}^{\epsilon K}=$ $\exp \left[-\mathcal{F}(K) / k_{B} T\right]$, where $\mathcal{F}(K)$ is the free energy at given $K$. It is convenient to redefine the fugacity $w$ as $w \rightarrow \xi w$, (as well as $w_{c} \rightarrow \xi w_{c}$ ) where $\xi$ is an arbitrary complex variable. Then the probability $P(K)$ can be found as the coefficient of $\xi^{K}$ in the function $\Xi_{N}(\xi w)$ expansion in powers of $\xi$. Therefore

$P(K)=\exp \left[-\mathcal{F}(K) / k_{B} T\right]=\frac{1}{2 \pi i} \oint \frac{\exp \left[-N \ln z^{*}(\xi w)\right]}{\xi^{K+1}} \mathrm{~d} \xi$,

where the contour of integration is a closed path in the complex $\xi$ plane around $\xi=0$ (see, e.g., [25]). To estimate the integral in eq. (20) we use the steepest-descent method [25].

For large $N$ the main contribution to the integral in eq. (20) is given by the saddle point $\xi=\xi_{0}$ of the integrand which is defined by the extremum of the function $g(\xi)=$ $-\ln z^{*}(\xi w)-[(K+1) / N] \ln \xi$, i.e., by the condition

$$
\frac{K+1}{\xi_{0}}=-\left.N \frac{\partial \ln z^{*}}{\partial \xi}\right|_{\xi=\xi_{0}} .
$$

The integral is dominated by the term $\exp \left[-N \ln z^{*}\right.$ $\left.\left(\xi_{0} w\right)-(K+1) \ln \xi_{0}\right]$. Another contribution comes from the integration along the steepest-descent line. As a result, one obtains

$$
P(K) \propto \frac{\exp \left[-N \ln z^{*}\left(\xi_{0} w\right)-(K+1) \ln \xi_{0}\right]}{\sqrt{N\left[\left(\frac{N}{K}\right)\left[\frac{\partial \ln z^{*}}{\partial \xi}\right]_{\xi=\xi_{0}}^{2}-\left.\frac{\partial^{2} \ln z^{*}}{\partial \xi^{2}}\right|_{\xi=\xi_{0}}\right]}} .
$$

The validity of the steepest-descent method is ensured by the condition of the large second derivative $N g^{\prime \prime}\left(\xi_{0}\right)$, which yields

$N g^{\prime \prime}\left(\xi_{0}\right)=N\left[\left(\frac{N}{K}\right)\left[\frac{\partial \ln z^{*}}{\partial \xi}\right]_{\xi=\xi_{0}}^{2}-\left.\frac{\partial^{2} \ln z^{*}}{\partial \xi^{2}}\right|_{\xi=\xi_{0}}\right] \gg 1$

A more explicit calculation whithin this method can be performed in the vicinity of the critical point $\epsilon=\epsilon_{c}$.

\subsection{PDF of the number of adsorbed monomers close to the critical point of adsorption}

In this case the explicit form of $\ln z^{*}$ is known [14] and after the redefinition of the fugacity, $w \rightarrow \xi w$, reads

$$
\ln z^{*}(\xi w)=-a_{1}\left(w-w_{c}\right)^{1 / \phi} \xi^{1 / \phi}-\ln \mu_{3},
$$

where $a_{1}$ is a constant of the order of unity. The critical adsorption fugacity $w_{c}=\exp \left(\epsilon_{c}\right)$ is defined by the equation

$$
\zeta(\alpha) \Phi\left(1-\gamma_{d=2}, \mu_{2} w_{c} / \mu_{3}\right)=1,
$$

with $\zeta(x)$ denoting the Riemann zeta-function.

By using eq. (24) in eq. (21), one arrives at the expression for the saddle point

$$
\xi_{0}=\left(\frac{K}{N}\right)^{\phi} \frac{a_{2}}{w-w_{c}},
$$

where $a_{2}=\left(\phi / a_{1}\right)^{\phi}$. Using eq. (24) and eq. (26) in eq. (22) then yields the expression for PDF

$$
P(K) \propto\left[\left(w-w_{c}\right) \mathrm{e}^{a_{2}}\right]^{K}\left(\frac{K}{N}\right)^{-\phi K-1 / 2} \frac{\mu_{3}^{N}}{N^{1 / 2}} .
$$

For reasonably large $K$ and after normalization, one arrives at the final expression for the PDF:

$$
P(K)=\frac{\eta^{K}}{\mathcal{C}(\eta) K^{\phi K}}
$$

where we have introduced the usual adsorption scaling variable $\eta=b_{1}\left(w-w_{c}\right) N^{\phi}\left(b_{1}\right.$ is a constant of the order of unity; see, e.g., [26]) as well as the normalization constant $\mathcal{C}$ :

$$
\mathcal{C}(\eta)=\sum_{K=1}^{N} \frac{\eta^{K}}{K^{\phi K}}
$$

One can readily see that the width of the distribution increases with $w$ or with $\epsilon$. To this end, one may directly calculate the variance of fluctuation as follows:

$\overline{(K-\bar{K})^{2}}=-N \frac{\partial^{2} \ln z^{*}(w)}{\partial(\ln w)^{2}} \propto N w\left(w / \phi-w_{c}\right)\left(w-w_{c}\right)^{1 / \phi-2}$,

where the expression for $\ln z^{*}(w)$ given by eq. (24) (where also $\xi=1$ ) has been used. Taking into account that $\phi \approx$ 0.5 , it becomes clear that the variance really grows with $w$.

The validity of the steepest-descent method is ensured by the condition eq. (23). Using eq. (24) and eq. (26), this criterion holds when $N\left(w-w_{c}\right)^{2}(K / N)^{1-2 \phi} \gg 1$. We recall that $\phi \approx 0.5$, so that $\left(w-w_{c}\right) N^{1 / 2} \gg 1$. As a result, the criterion becomes

$$
N^{-1 / 2} \ll\left(w-w_{c}\right) \ll 1 .
$$

In the deep adsorption regime this condition might be violated. Nevertheless, the steepest-descent method still could be used there provided that the appropriate solution for $z^{*}(w)$ (see eq. (10)) is chosen. 


\subsection{The regime of deep adsorption}

In the deep adsorption regime we should use the solution for $z^{*}(w)$ which was also discussed in ref. [14]. Namely, in this case

$$
z^{*}(w) \approx \frac{1}{\mu_{2} w}\left[1-\left(\frac{\mu_{3}}{\mu_{2} w}\right)^{1 /(1-\lambda)}\right]
$$

With eq. (32) the mean value $\bar{K}=-N \partial \ln z^{*}(w) / \partial \ln w$ can be written as

$$
\frac{\bar{K}}{N}=1-\frac{1}{1-\lambda}\left(\frac{\mu_{3}}{\mu_{2} w}\right)^{1 /(1-\lambda)},
$$

thus $\bar{K}$ tends to $N$ with $w$ growing as it should be. The variance of the fluctuations within the GCE then becomes

$$
\overline{(K-\bar{K})^{2}}=-N \frac{\partial^{2} \ln z^{*}(w)}{\partial(\ln w)^{2}}=\frac{N}{(1-\lambda)^{2}}\left(\frac{\mu_{3}}{\mu_{2} w}\right)^{1 /(1-\lambda)},
$$

i.e., the fluctuations decrease when the adsorption energy grows. Comparison of this result with the result given by eq. (30) leads to the important conclusion that the fluctuations of the number of adsorbed monomers first grow with $\epsilon$, attain a maximum, and finally decrease with increasing surface adhesion $\epsilon$. The position of the maximum reflects the presence of finite-size effects, however, as the chain length $N \rightarrow \infty$, this maximum occurs at the CAP.

Consider now the steepest-descent treatment for the deep adsorption regime. With eq. (32) (after the rescaling $w \rightarrow \xi w)$ in eq. (21), the saddle point becomes

$$
\xi_{0}=b_{2}\left(\frac{\mu_{3}}{\mu_{2} w}\right)\left(1-\frac{K}{N}\right)^{-(1-\lambda)}
$$

where $b_{2}=(1-\lambda)^{-(1-\lambda)}$ (recall that $1-\lambda=\gamma_{d=2}=$ 1.343). The main contribution comes from the exponential term in eq. (22) which is given by

$$
\begin{gathered}
\exp \left[-N \ln z^{*}\left(w \xi_{0}\right)-(K+1) \ln \xi_{0}\right]= \\
\left(\mu_{2} w\right)^{N} \frac{\left(b_{2} \mu_{3} \mathrm{e}^{1-\lambda} N^{1-\lambda} / \mu_{2} w\right)^{N-K}}{(N-K)^{(1-\lambda)(N-K)}} .
\end{gathered}
$$

For $N-K \gg 1$ the expression for the non-normalized PDF takes the form

$$
P(K) \propto \frac{\left(b_{3} \mu_{3} N^{1-\lambda} / \mu_{2} w\right)^{N-K}}{(N-K)^{(1-\lambda)(N-K)}},
$$

where $b_{3}$ is a constant of the order of unity. Normalization of this distribution yields

$$
P(K)=\frac{\chi^{N-K}}{\mathcal{R}(\chi)(N-K)^{(1-\lambda)(N-K)}},
$$

where the parameter $\chi=b_{3}\left(\mu_{3} / \mu_{2} w\right) N^{1-\lambda}$ and the normalization constant

$$
\mathcal{R}(\chi)=\sum_{K=1}^{N-1} \frac{\chi^{N-K}}{(N-K)^{(1-\lambda)(N-K)}} .
$$
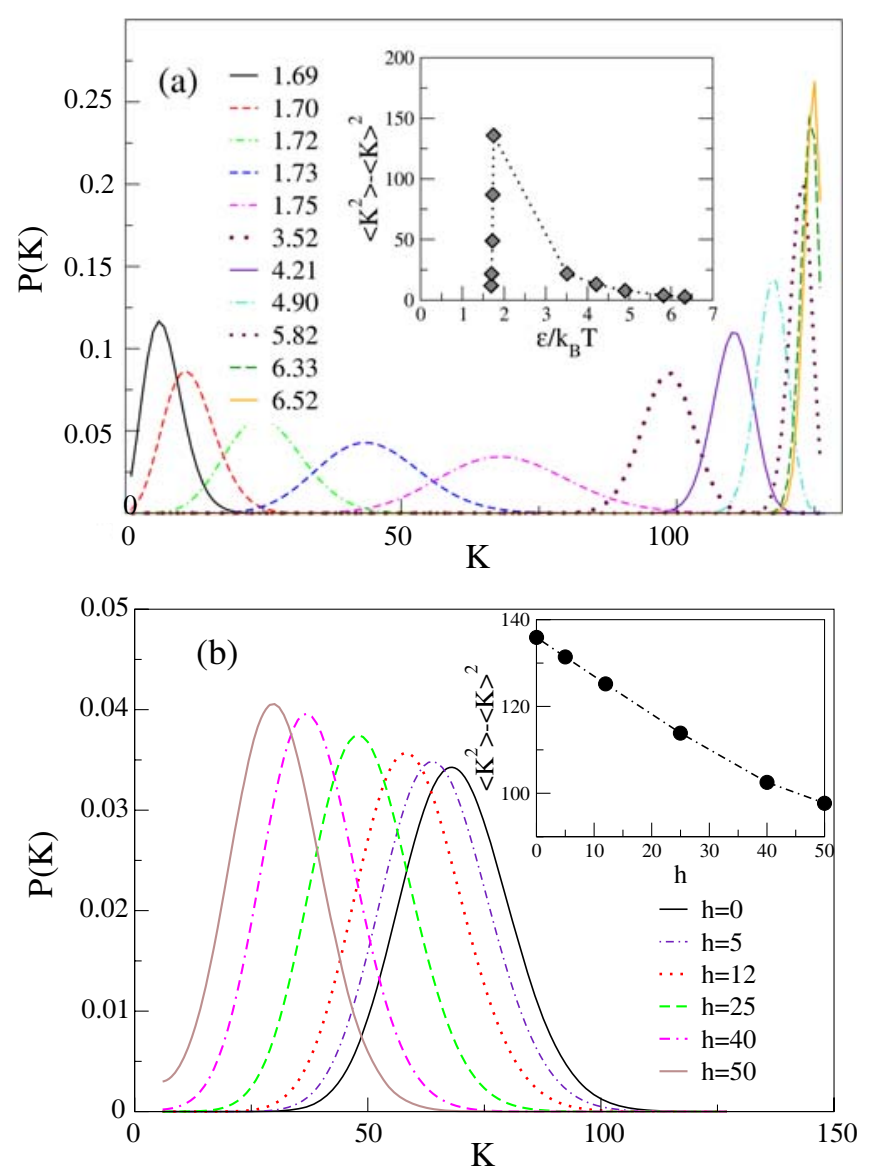

Fig. 3. (a) Probability distribution function of the number of chain contacts with the grafting plane, $K$, for different degrees of adhesion $\epsilon$ and no pulling force $f=0$ as follows from eqs. (28) and (38). The variance of $P(K)$ is shown in the inset for different $\epsilon$. (b) The same as in (a) for $\epsilon=1.75$ and different heights of the chain-end $h$. The change of the variance $\left\langle K^{2}\right\rangle-\langle K\rangle^{2}$ with $h$ is shown in the inset.

The validity condition, eq. (23), in the deep adsorption regime (after substitution of eq. (32) into eq. (23)) requires

$$
(N-K)^{3 / 2-\lambda} \gg\left(\mu_{3} / \mu_{2} w\right) N^{1-\lambda},
$$

i.e., $K$ should not be very close to $N$. In fig. 3 a we show the PDF of the number of chain contacts, $P(K)$, for a free chain without pulling and several adsorption strengths of the substrate. One can readily verify that visually the shape of $P(K)$ resembles very much a Gaussian distribution for moderate values of $\epsilon_{c} \approx 1.7<\epsilon<6.0$. The $\mathrm{PDF}$ variance goes through a sharp maximum at $\epsilon \gtrsim \epsilon_{c}$ and then declines, as expected from eq. (34).

One should note that in the $f$ ensemble (where the force $f$ and not the distance $h$ acts as a control parameter [14]) the order parameter $n=\bar{K} / N$ undergoes a jump at the detachment adsorption energy $\epsilon_{D}$. This means that $N\left(\partial^{2} \ln z^{*} / \partial \epsilon^{2}\right)_{\epsilon_{D}}=-(\partial \bar{K} / \partial \epsilon)_{\epsilon_{D}} \rightarrow-\infty$. Thus at the detachment point the variance of the fluctuations $\overline{(K-\bar{K})^{2}}=-N\left(\partial^{2} \ln z^{*} / \partial \epsilon^{2}\right)_{\epsilon_{D}} \rightarrow \infty$, which practically means that for chains of a finite length the 
distribution at $\epsilon=\epsilon_{D}$ becomes very broad, in sharp contrast to eq. (34). This has indeed been observed in our MC simulation results (see fig. 12 in ref. [14]).

\section{3 $\mathrm{P}(\mathrm{K})$ distribution in the subcritical regime $\mathrm{w}<\mathrm{w}_{\mathrm{c}}$ of underadsorption}

In the subcritical regime, $w<w_{c}$, the fraction of adsorbed points (order parameter) $n=\bar{K} / N=0$, in the thermodynamic limit. Nevertheless, $\bar{K} \neq 0$ and one can pose a question about the form of $\operatorname{PDF} P(K)$. At $w<w_{c}$ the solution for $z^{*}(w)$ (the simple pole of $\Xi(z, w)$ in the complex $z$-plane) does not exist because $V(w z) U(z)<1$ (see eq. (18)). However, the tail GCE partition function $Q(z)=1+\Phi\left(1-\gamma_{1}, \mu_{3} z\right) \propto \Gamma\left(\gamma_{1}\right) /\left(1-\mu_{3} z\right)^{\gamma_{1}}$ has a branch point at $z=1 / \mu_{3}$ (see eq. (A 11) in ref. [14]) which governs the coefficient at $z^{N}$, i.e., the partition function $\Xi_{N}(w)$. The calculation (following sect. 2.4.3 in ref. [25]) yields

$$
\Xi_{N}(w)=\frac{1+\Phi\left(\lambda, \mu_{2} w / \mu_{3}\right)}{1-\zeta(\alpha) \Phi\left(\lambda, \mu_{2} w / \mu_{3}\right)} \mu_{3}^{N} N^{\gamma_{1}-1}
$$

where $\lambda=1-\gamma_{d=2}$ and we have also used that at $z=1 / \mu_{3}$ the loop and train GCE partition functions are $U\left(1 / \mu_{3}\right)=$ $\Phi(\alpha, 1)=\zeta(\alpha)$ and $V\left(w / \mu_{3}\right)=\Phi\left(\lambda, \mu_{2} w / \mu_{3}\right)$, respectively. This expression has a pole at $w=w_{c}$ (cf. eq. (25)) which yields the coefficient of $w^{K}$, i.e. $\Xi_{N, K}$. Recall that $\Xi_{N}(w)=\sum_{K=0}^{\infty} \Xi_{N, K} w^{K}$, so that $P(K) \propto \Xi_{N, K} w^{K}$. Expansion of the denominator in eq. (41) around $w=w_{c}$ reveals the simple pole as follows:

$$
\Xi_{N}(w)=\frac{[1+1 / \zeta(\alpha)] \mu_{3}^{N} N^{\gamma_{1}-1}}{\zeta(\alpha) \Phi\left(-\gamma_{d=2}, \mu_{2} w_{c} / \mu_{3}\right)}\left(\frac{w_{c}}{w_{c}-w}\right) .
$$

In (42) we have used the relationship $x(\mathrm{~d} / \mathrm{d} x) \Phi(1-$ $\left.\gamma_{d=2}, x\right)=\Phi\left(-\gamma_{d=2}, x\right)$. The coefficient of $w^{K}$, i.e., $\Xi_{N, K}$, is proportional to $w_{c}^{-K}$. Therefore $P(K) \propto\left(w / w_{c}\right)^{K}=$ $\exp \left[-\left(\epsilon_{c}-\epsilon\right) K\right]$. Taking the normalization condition $\sum_{K=0}^{N} P(K)=1$ into account, the final expression for $P(K)$ can be recast in the form

$$
P(K)=\frac{1-\exp \left[-\left(\epsilon_{c}-\epsilon\right)\right]}{1-\exp \left[-N\left(\epsilon_{c}-\epsilon\right)\right]} \exp \left[-K\left(\epsilon_{c}-\epsilon\right)\right],
$$

i.e., $P(K)$ has a simple exponential form. The calculation of the average $\bar{K}=\sum_{K=0}^{N} K P(K)$ leads to the simple result $\bar{K}=\left[\exp \left(\epsilon_{c}-\epsilon\right)-1\right]^{-1} \approx 1 /\left(\epsilon_{c}-\epsilon\right)$, i.e., $\bar{K} \rightarrow \infty$ at $\epsilon \rightarrow \epsilon_{c}$. On the other hand, we know that $\bar{K}=N^{\phi}$ at $\epsilon=\epsilon_{c}$. In order to prevent a divergency at $\epsilon=\epsilon_{c}$, one should incorporate an appropriate cutoff in the PDF given by eq. (43). With this the distribution is given by

$$
\begin{aligned}
P(K)= & \frac{1-\exp \left[-\left(\epsilon_{c}-\epsilon+1 / N^{\phi}\right)\right]}{1-\exp \left[-N\left(\epsilon_{c}-\epsilon+1 / N^{\phi}\right)\right]} \\
& \times \exp \left[-K\left(\epsilon_{c}-\epsilon+1 / N^{\phi}\right)\right] .
\end{aligned}
$$

Thus the expression for the average number of adsorbed monomer has the correct limit behavior, i.e.,

$$
\bar{K}=\frac{1}{\epsilon_{c}-\epsilon+1 / N^{\phi}} .
$$

\subsection{Probability distribution function $P(K)$ in the h-ensemble}

Eventually we examine how the fixed chain-end height $h$ affects the PDF of the number of contacts $K$. To this end, we refer again to fig. 2a where an adsorbed chain with a fixed last monomer height $h$ is depicted. The adsorbed chain consists of a tail of length $M$ and of an adsorbed part with $N-M$ beads. One should bear in mind that $M$ is a function of the control parameters $h$ and $w=\exp (\epsilon)$ given by eqs. (12) and (13). The partition function of the adsorbed part is then given by

$\Xi_{N}(w)=\sum_{K=0}^{N} \Xi_{N, K} w^{K}=\exp \left\{-[N-M(h, w)] \ln z^{*}(w)\right\}$,

where we took into account that the free energy of the adsorbed portion is given as $F_{\text {ads }}=k_{B} T[N-$ $M(h, w)] \ln z^{*}(w)$ (see sect. 2.2).

As mentioned above, the PDF $P(K) \propto \Xi_{N, K} w^{K}$, so that by means of rescaling $w \rightarrow \xi w$ and $w_{c} \rightarrow \xi w_{c}$ the PDF can be found as the coefficient of $\xi^{K}$, i.e.,

$$
P(K)=\frac{1}{2 \pi i} \oint \frac{\exp \left\{-[N-M(h, \xi w)] \ln z^{*}(\xi w)\right\}}{\xi^{K+1}} \mathrm{~d} \xi .
$$

As well as before, the steepest-descent method can be used to calculate the integral in eq. (47). However, in this case the calculations are more complicated and we have relegated most of them to appendix B. As may be seen there, the saddle point equation cannot be solved analytically in general case but could be treated iteratively for relatively small heights $h$.

One can readily see that for $h=0$, eq. (B.7) reduces to eq. (28). The PDF, following from eq. (B.7) is shown in fig. $3 \mathrm{~b}$ for $\epsilon=1.75$ and several values of the height $h$. It can be seen that the curve for $h=0$ coincides with the curve for $\epsilon=1.75$ in fig. 3a as it should be. Evidently, both the mean value $\bar{K}$ and $\overline{K^{2}}-\bar{K}^{2}$ decline with growing $h$.

\section{Monte Carlo simulation model}

We use a coarse-grained off-lattice bead-spring model [27] which has proved rather efficient in a number of polymers studies so far. The system consists of a single polymer chain tethered at one end to a flat impenetrable structureless surface. The surface interaction is described by a square well potential,

$$
U_{w}(z)= \begin{cases}\epsilon, & z<r_{c} \\ 0, & z \geq r_{c}\end{cases}
$$

The strength $\epsilon$ is varied from 2.0 to 5.0 while the interaction range $r_{c}=0.125$. The effective bonded interaction is described by the FENE (finitely extensible nonlinear elastic) potential:

$$
U_{\mathrm{FENE}}=-K\left(1-l_{0}\right)^{2} \ln \left[1-\left(\frac{l-l_{0}}{l_{\max }-l_{0}}\right)^{2}\right],
$$


with $K=20, l_{\max }=1, l_{0}=0.7, l_{\min }=0.4$. The nonbonded interactions between monomers are described by the Morse potential:

$$
\frac{U_{M}(r)}{\epsilon_{M}}=\exp \left(-2 \alpha\left(r-r_{\min }\right)\right)-2 \exp \left(-\alpha\left(r-r_{\min }\right)\right),
$$

with $\alpha=24, r_{\min }=0.8, \epsilon_{M} / k_{B} T=1$. In few cases, needed to clarify the nature of the polymer chain resistance to stretching, we have taken the non-bonded interactions between monomers as purely repulsive by shifting the Morse potential upward by $\epsilon_{M}$ and removing its attractive branch, $V_{M}(r)=0$ for $r \geq r_{\text {min }}$.

We employ periodic boundary conditions in the $x-y$ directions and impenetrable walls in the $z$ direction. The lengths of the studied polymer chains are typically 64 , and 128. The size of the simulation box was chosen appropriately to the chain length, so for example, for a chain length of 128 , the box size was $256 \times 256 \times 256$. All simulations were carried out for constant position of the last monomer $z$-coordinate, that is, in the fixed height ensemble. The fluctuating force $f$, exerted on the last bead by the rest of the chain, was measured and average over about 2000 measurements. The standard Metropolis algorithm was employed to govern the moves with self-avoidance automatically incorporated in the potentials. In each Monte Carlo update, a monomer was chosen at random and a random displacement attempted with $\Delta x, \Delta y, \Delta z$ chosen uniformly from the interval $-0.5 \leq \Delta x, \Delta y, \Delta z \leq 0.5$. If the last monomer was displaced in $z$ direction, there was an energy cost of $-f \Delta z$ due to the pulling force. The transition probability for the attempted move was calculated from the change $\Delta U$ of the potential energies before and after the move was performed as $W=\exp \left(-\Delta U / k_{B} T\right)$. As in a standard Metropolis algorithm, the attempted move was accepted, if $W$ exceeds a random number uniformly distributed in the interval $[0,1]$.

As a rule, the polymer chains have been originally equilibrated in the MC method for a period of about $5 \times 10^{5}$ MCS after which typically 500 measurement runs were performed, each of length $2 \times 10^{6} \mathrm{MCS}$. The equilibration period and the length of the run were chosen according to the chain length and the values provided here are for the longest chain length.

\section{Monte Carlo simulation results}

In order to verify the theoretical predictions, outlined in sect. 2, we carried out extensive Monte Carlo simulations with the off-lattice model, defined in sect. 4 . In these simulations we fix the end monomer of the polymer chain at height $h$ above the adsorbing surface, and measure the (fluctuating) force, needed to keep the last bead at distance $h$, as well as the corresponding fraction of adsorbed monomers $n$. These computer experiments are performed at different strengths $\epsilon$ of the adsorption potential, eq. (48). In figs. 4a,b we compare the predicted dependence of the order parameter $n$ on the (dimensionless) height $h / l_{0} N$ at several values of $2.0 \leq \epsilon \leq 5.0$ with the
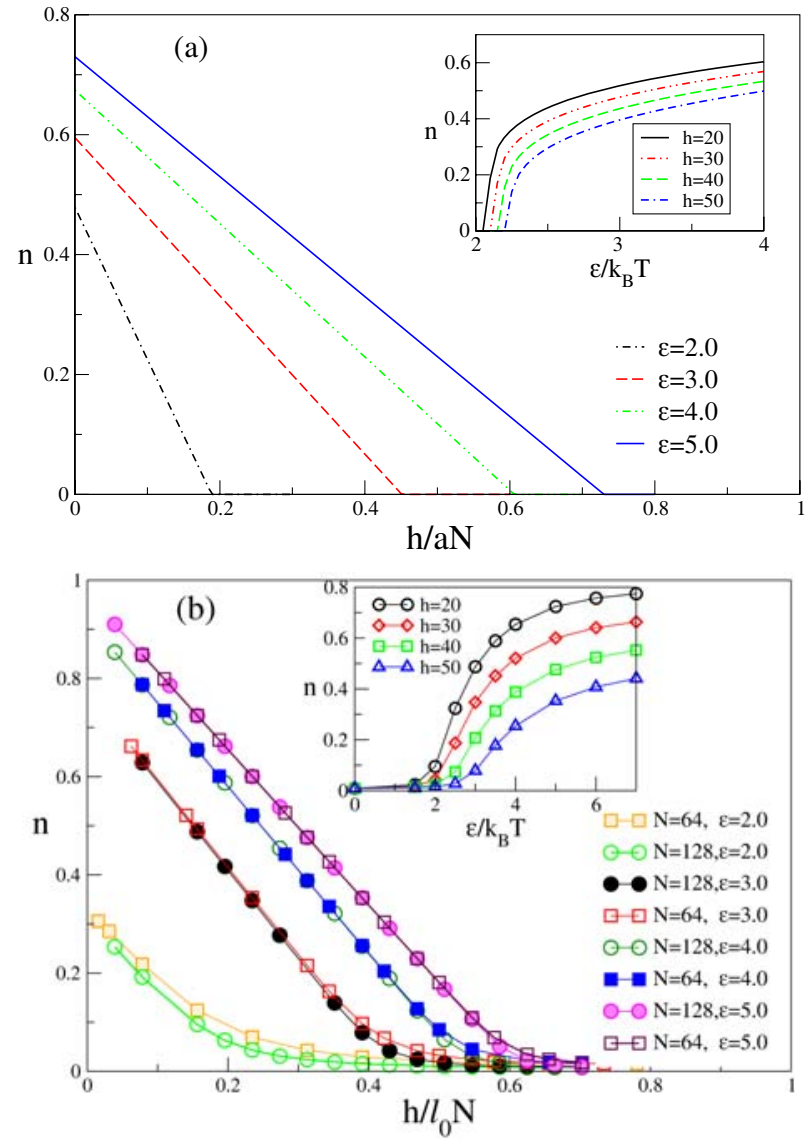

Fig. 4. (a) Order parameter (fraction of adsorbed monomers) $n$ variation with changing height $h / l_{0} N$ of the fixed chainend for polymers of length $N=128$ and different adsorption strength $\varepsilon / k_{B} T$. (b) Variation of $n$ with $\varepsilon / k_{B} T$ for different fixed positions of the chain-end $h / l_{0} N$ as is seen from MC data. Insets show the resulting $n-\epsilon$ relationship at several fixed heights $h$.

results from MC simulations. Note that the critical point of adsorption $\epsilon_{c} \approx 1.69$, so we take our measurements above the region of critical adsorption. Typically, both in the analytic results, fig. $4 \mathrm{a}$, and in the MC data, fig. $4 \mathrm{~b}$, for $N=128$, one recovers the predicted linear decrease of $n$ with growing $h$. Finite-size effects lead to some rounding of the simulation data (in fig. $4 \mathrm{~b}$ these effects are seen to be larger for $N=64$ than for $N=128$ ) when $n \rightarrow 0$ so that the height of detachment $h_{D}$ is determined from the intersection of the tangent to $n(h)$ and the $x$-axis where $n=0$. Evidently, with growing adsorption strength, $\epsilon$, a larger height $h_{D}$ is needed to detach the polymer from the substrate. Thus, one may construct a phase diagram for the desorption transition, which we show in fig. 5. The theoretical prediction is given by eq. (17).

In the insets of fig. $4 \mathrm{a}, \mathrm{b}$ we also show the variation of the fraction of adsorbed segments $n$ with adsorption strengths $\epsilon$ for several heights $20 \leq h \leq 50$ of a chain with $N=128$. It is evident that, apart from the rounding of the MC data for $n$ at $n \rightarrow 0$, one finds again a pretty good agreement between the behavior, predicted by eq. (15), and the simulation results. 


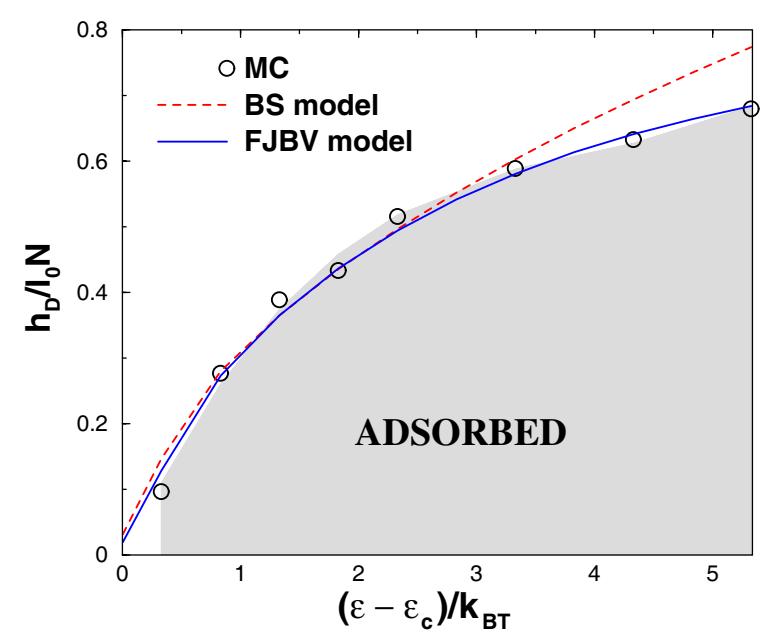

Fig. 5. Phase diagram showing the dependence of the critical height of polymer detachment from the substrate, $h_{D} / l_{0} N$, with the relative strength of adsorption $\left(\varepsilon-\varepsilon_{c}\right) / k_{B} T$, where $\varepsilon_{c} / k_{B} T$ is the critical point of adsorption at zero force. The theoretical curves follow eq. (17).

The gradual change of $n$ in the whole interval of possible variation of $h$ suggests a pseudo-continuous phase transition, as pointed out in the end of sect. 2, eq. (16). Of course, if $h$ is itself expressed in terms of the measured pulling force, one would again find that $n$ changes abruptly with varying $f$ at some threshold value $f_{D}$, indicating a first-order transformation from an adsorbed into a desorbed state of the polymer chain.

It has been pointed out earlier by Skvortsov et al. [12] that while both the fixed-force and the fixed-height ensembles are equivalent as far as the mean values of observables such as the fraction of adsorbed monomers and other related quantities are concerned, this does not apply to some more detailed properties like those involving fluctuations. Therefore, it is interesting to examine the fluctuations of the order parameter, $n$, for different values of our control parameter $h$, and compare them to theoretical predictions for $P(K)$ from sect. 2. First we compare the order parameter distribution $P(n)$ for zero force, fig. 6 , obtained from our computer experiment, to that, predicted by eqs. $(28,38,44)$, and displayed in fig. $3 \mathrm{a}$. It is evident from fig. 6 that for free chains at different strengths of adhesion there is a perfect agreement between analytical and simulational results. For rather weak adsorption $\epsilon=1.3 \pm 1.6<\epsilon_{c}=1.67$ in the subcritical regime, one can verify from fig. 6 that $P(n)$ gradually transforms from nearly Gaussian into exponential distribution, as expected from eq. (44). For $\epsilon>\epsilon_{c}$ the distribution width grows and goes through a sharp maximum in the vicinity of $\epsilon_{c}$, and then drops as $\epsilon$ increases further - compare insets in fig. 6 and fig. 3a.

Let us consider now PDF in the presence of pulling. In fig. 7a we display the distribution $P(n)$ measured in the MC simulations for different heights $h$ and constant adsorption energy $\epsilon=3.0$. One can readily verify from our results that far enough from the detachment line, $h<h_{D}$,

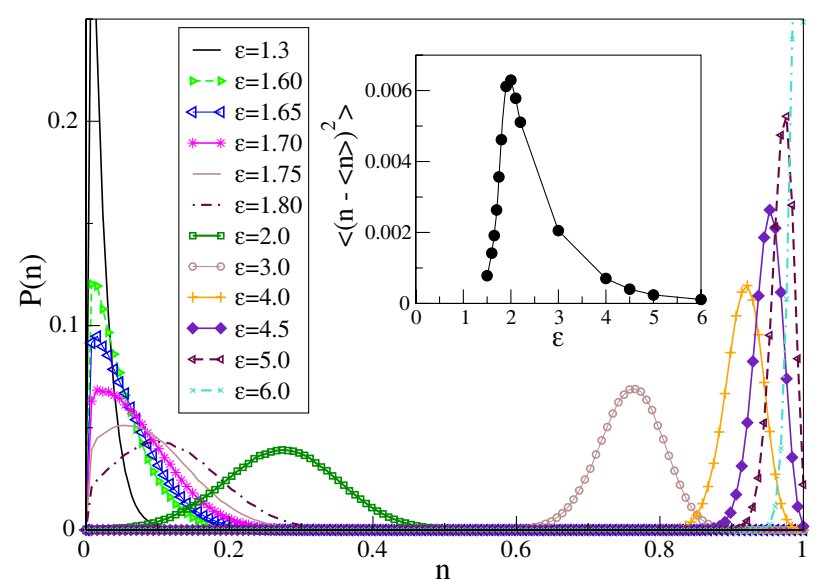

Fig. 6. PDF of the order parameter (fraction of contacts with the plane) for different adsorption strength $\epsilon$ at zero force. The critical adsorption point (CAP) $\epsilon_{c} \approx 1.67$. The change of the variance $\left\langle n^{2}\right\rangle-\langle n\rangle^{2}$ with varying $\epsilon$ is displayed in the inset.

the shape of $P(n)$ looks like Gaussian and that the second moment, $\left\langle(\Delta n)^{2}\right\rangle$, remains unchanged with varying height $h$. Of course, when $h \rightarrow h_{D}$, the maximum of $P(n)$ shifts to lower values of $n$. Only in the immediate vicinity of $h_{D}$, where $n \rightarrow 0$ and the fluctuations strongly decrease, one observes a significant deviation from the Gaussian shape - cf. the inset in fig. 7a. The latter is illustrated in more detail in fig. $7 \mathrm{~b}$ where we show the measured variation of the second moment, $\left\langle(\Delta n)^{2}\right\rangle$, and that of the third moment, $\left\langle(n-\langle n\rangle)^{3}\right\rangle$ with increasing height $h$. The deviation from Guassinity in $P(n)$, measured by the deviation of the third moment from zero, is localized in the vicinity of the detachment height $h_{D}$. The corresponding theoretical prediction for the relatively weak adsorption strength is depicted in fig. 3b. It can be seen that with increasing $h$ the almost Gaussian distribution tends to a Poisson-like one. Also the fluctuations decrease with $h$ in accordance with MC findings.

The force $f$, exerted by the chain on the end-monomer, when the latter is kept at height $h$ above the surface, is one of the main properties which can be measured in experiments carried out within the fixed-height ensemble. Note that $f$ has the same magnitude and opposite sign, regarding the force, applied by the experimentalist. The variation of the force $f$ with increasing height $h$ is shown in fig. 8a for several values of the adsorption potential $2.0 \leq \epsilon \leq 5.0$. In fig. 8a we distinguish between two contributions in the total force $f$, acting on the end bead. The first stems from the quasi-elastic forces of the bonded interaction (FENE) whereas the second contribution is due to the short-range (attractive) interactions between nonbonded monomers (in our model the Morse potential). A typical feature of the $f$ - $h$ relationship, namely, the existence of a broad interval of heights $h$ where the force remains constant (a plateau in the force) is readily seen in fig. 8a. With growing strength of adsorption $\epsilon$ the length of this plateau as well as the magnitude of the plateau force increase. Note that, for $\epsilon=0$, no plateau whatsoever is found. Upon further extension (by increasing $h$ ) 

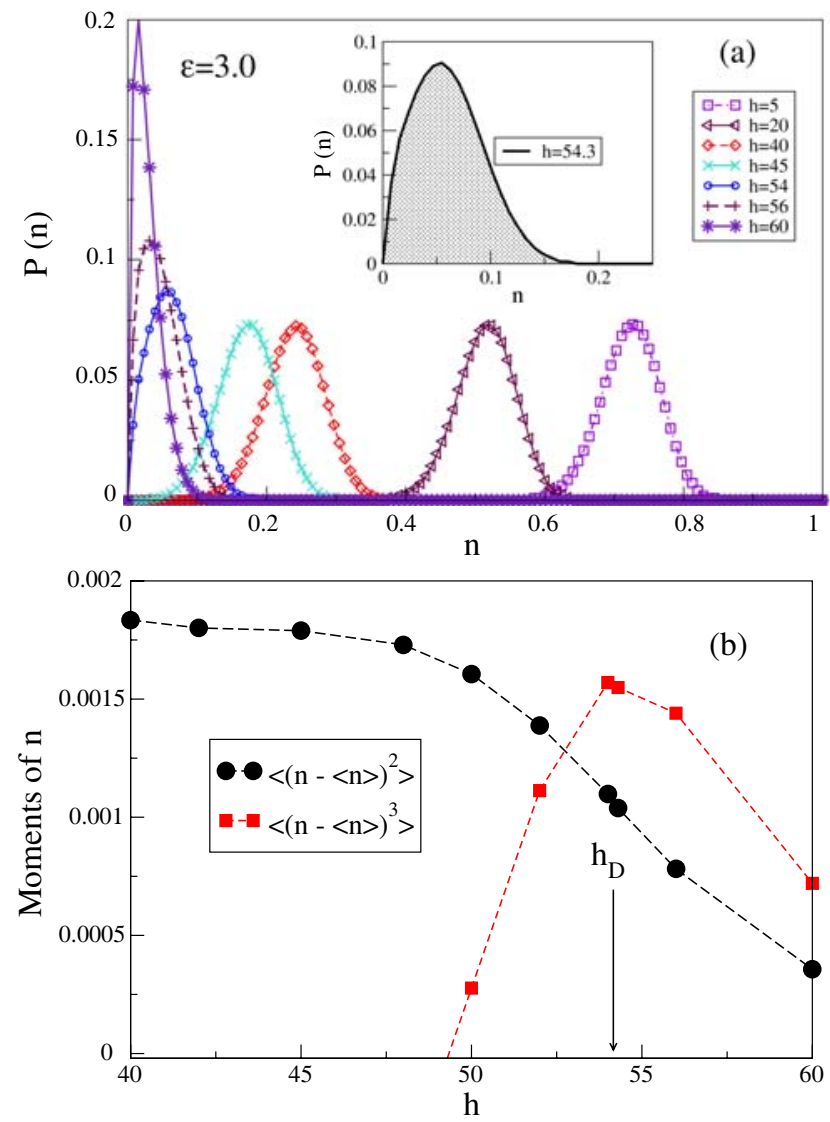

Fig. 7. (a) Probability distribution $P(n)$ of the order parameter $n$ (i.e., the fraction of adsorbed monomers) for $N=128$ and $\epsilon=3.0$ at different heights of the chain-end $h$ over the grafting plane. In the inset we show $P(n)$ at the detachment line $h_{D}=54.3$. (b) Variation of the second and third central moments of $P(n)$ with $h$. The maximum of $\left\langle(n-\langle n\rangle)^{3}\right\rangle$ is reached at $h=h_{D}$.

of the chain, the plateau ends and the measured force starts to grow rapidly in magnitude - an effect, caused by a change of the chain conformation itself in the entirely desorbed state.

A closer inspection of fig. 8a reveals that the nonbonded contribution to $f$, which is generally much weaker than the bonded one, behaves differently, depending on whether the forces between non-nearest neighbors along the backbone of the chain are purely repulsive, or contain an attractive branch. While for strong adsorption, $\epsilon \geq 3.0$, a plateau is observed even for attractive non-bonded interactions, for weak adsorption, $\epsilon \leq 2.0$, an increase of the non-bonded contribution at $h / \bar{l}_{0} N \approx 0.35$, (seen as a minimum in fig. 8a) is observed. This effect is entirely missing in the case of purely repulsive non-bonded interactions - see the inset in fig. 8a where the contributions from bonded and non-bonded interactions are shown for a neutral surface $\epsilon=0$. If one plots the magnitude of the measured force at the plateau against the corresponding value of the adsorption potential, $\epsilon$, one may check the theoretical result, eq. (12) - fig. 8b. Evidently, the theoretical predictions about $f_{\mathrm{p}}$ agree well with the observed
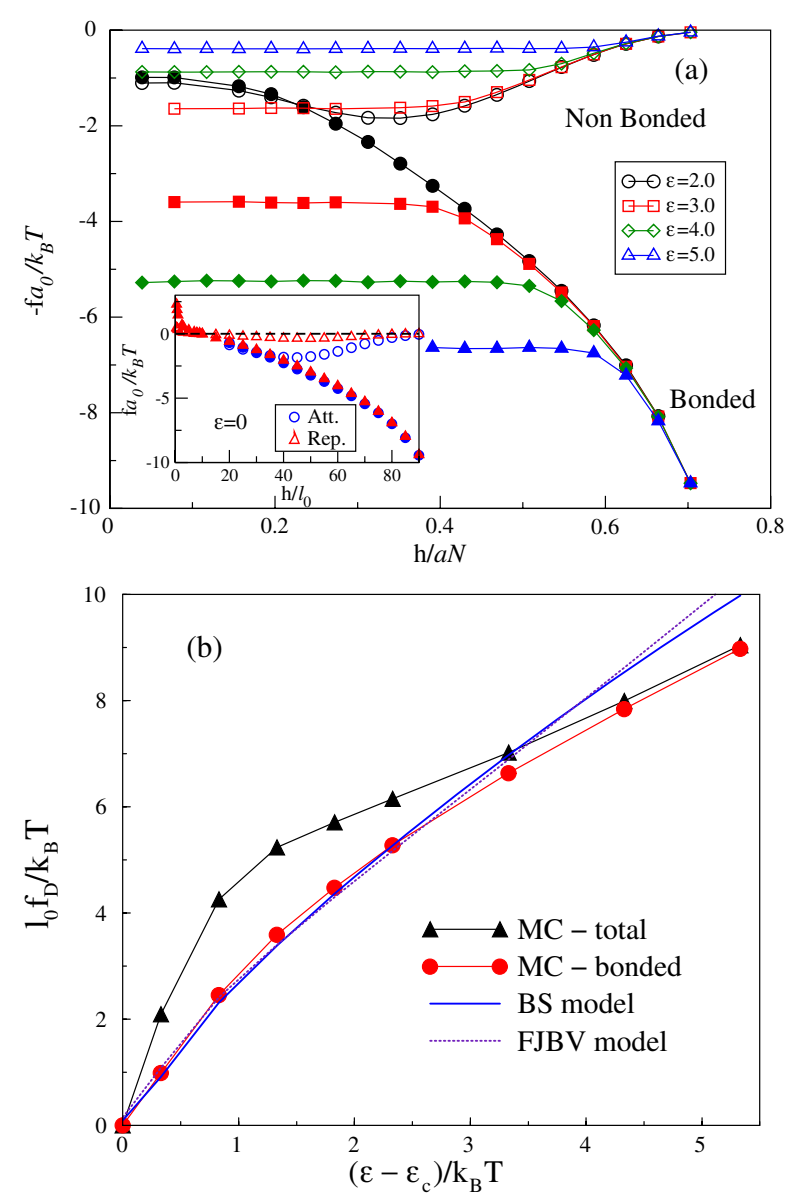

Fig. 8. (a) Variation of the two components to the total force, exerted by the chain on the end-monomer which is fixed at a (dimensionless) height $h / l_{0} N$ for different adsorption potentials $2.0 \leq \epsilon / k_{B} T \leq 5.0$ : bonding interactions (full symbols) and non-bonding Morse interactions (empty symbols). In the inset the same is shown for a neutral plane $\epsilon=0.0$ for purely repulsive monomers (triangles) and for such with a weak Morse attraction (circles). (b) Variation of the total force (plateau hight) exerted by the AFM tip on the chain-end for chain length $N=128$ with the relative strength of adsorption $\left(\varepsilon-\varepsilon_{c}\right) / k_{B} T$.

variation of the detachment force in the MC simulation, both within the BS or FJBV models, as long as only the excluded-volume interactions in the MC data are taken into account. If the total contribution to $f_{\mathrm{p}}$, including also attractive non-bonded interactions in the chain, is depicted - black triangles in fig. 8b - then the agreement with the theoretical curves deteriorates since the latter do not take into account the possible presence of attractive non-bonded interactions.

The $f$ - $h$ relationship, which gives the equation of state of the stretched polymer, may be derived within one of the different theoretical models, e.g., that of BS, eq. (6), or FJBV model, eq. (8), as mentioned in sect. 2. Which of these theoretical descriptions is the more adequate can be decided by comparison with experiment. In fig. 9a,b, we present such comparison by plotting our simulation data 

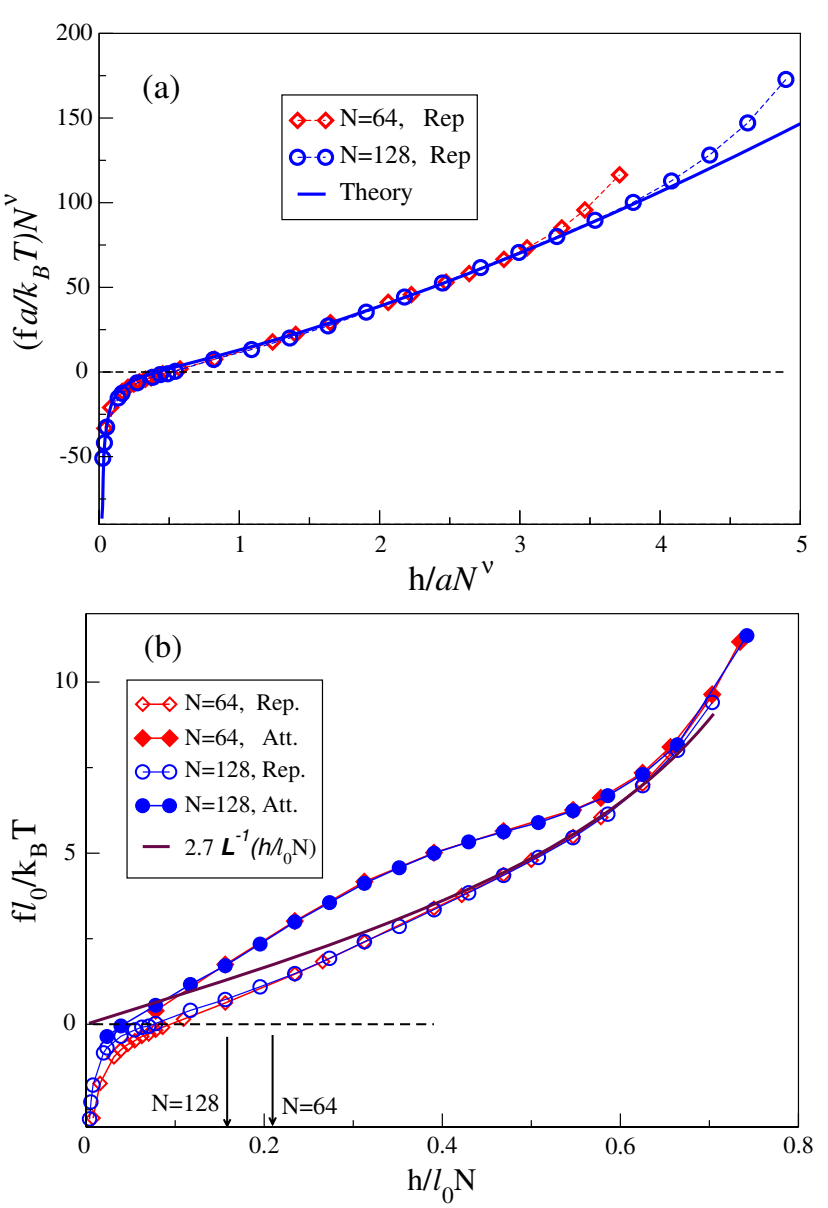

Fig. 9. (a) Variation of the total applied force $f$ with growing height of the end-monomer in terms of Pincus reduced variables, $f l_{0} N^{\nu} / k_{B} T$ versus $h / l_{0} N^{\nu}$, for a polymer with purely repulsive non-bonded forces for $N=64,128$. (b) The same as in (a) but in terms of reduced units $f l_{0} / k_{B} T$ versus $h / l_{0} N$ for purely repulsive (empty symbols) as well as for usual Morse potential (full symbols) of non-bonded interactions between monomers. The FJBV model results, eq. (8), is shown by a solid line. Arrows indicate the unperturbed gyration radius positions $R_{g} / N$ for $N=64,128$.

using different normalization for the height $h$. From fig. 9a it becomes evident that the data from our computer experiment for $N=64$ and $N=128$ collapse on a single curve, albeit this collapse only holds as long as $h / l_{0} N^{\nu} \leq 3.0$ for the BS model while it fails for stronger stretching. In contrast, this collapse works well for all values of $h$, provided the height is scaled with the contour length of the chain $N$, rather than with $N^{\nu}$, as in the FJBV model, fig. 9b, regardless of whether a purely repulsive, or the full Morse potential (which includes also an attractive part) of interactions is involved. The analytical expression, eq. (8), is found to provide perfect agreement with the simulation data for strong stretching, $h / l_{0} N \geq 0.4$. From the simulation data on fig. 9 one may even verify that the force $f$ goes through zero at some height $h>0$ and then turns negative, provided one keeps the chain-end very close to the grafting surface (cf. eq. (6)).

\section{Summary}

In the present work we have treated the force-induced desorption of a self-avoiding polymer chain from a flat structureless substrate both theoretically and by means of Monte Carlo simulation within the constant-height ensemble. The motivation for this investigation has been the necessity to distinguish between results obtained in this ensemble and results, derived in the constant-force ensemble, considered recently [14], as far as both ensembles could in principle be used by experimentalists. We demonstrate that the observed behavior of the main quantity of interest, namely, the fraction of adsorbed beads $n$ (i.e., the order parameter of the phase transition) with changing height $h$ differs qualitatively from the variation of the order parameter when the pulling force is varied. In the constant-height ensemble one observes a steady variation of $n$ with changing $h$, whereas in the constant-force ensemble one sees an abrupt jump of $n$ at a particular value of $f_{D}$, termed a detachment force. However, this should not cast doubts on the genuine first-order nature of the phase transition which can be recovered within the constantheight ensemble too, provided one expresses the control parameter $h$ in terms of the average force $f$. This equivalence has been studied extensively for Gaussian chains by Skvortsov et al. [12] who noted that ensemble equivalence does not apply to fluctuations of the pertinent quantities too.

Indeed, in our earlier study [13] we found diverging variance of the $\operatorname{PDF} P(n)$ at $f_{D}$ whereas in our present study the fluctuations of the order parameter are observed to stay finite at the transition height $h_{D}$. These findings confirm theoretical predictions based on analytic results which we derive within the GCE approach. Within this approach, we have explored two different theoretical models for the basic force-extension relationship, namely, the bead-spring (BS) model as well as that of a Freely Jointed Bond Vectors (FJBV) model. Our simulation results indicate a good agreement between theory and computer experiment.

We are indebted to A. Skvortsov for useful discussions during the preparation of this work. A. Milchev thanks the MaxPlanck Institute for Polymer Research in Mainz, Germany, for hospitality during his visit at the institute. A. Milchev and V.G. Rostiashvili acknowledge support from the Deutsche Forschungsgemeinschaft (DFG), grant No. SFB 625/B4.

\section{Appendix A. Freely jointed bond vectors model}

The deformation law in the overstretched regime (when the chain deformation is close to its saturation) could be treated better within the FJBV model. Let us consider a tethered chain of the length $N$ with one end anchored at the origin of the coordinates and the external force $f_{N}$ acting on the free end. The corresponding deformation 
energy reads

$$
U_{\text {ext }}=-f_{N} r_{N}^{\perp}=-f_{N} \sum_{i=1}^{N} b_{i} \cos \theta_{i}
$$

where $r_{N}^{\perp}$ is the $z$-coordinate (directed perpendicular to the surface) of the chain-end, $b_{i}$ and $\theta_{i}$ are the length and the polar angle of the $i$-th bond vector, respectively. The corresponding partition function of the FJBV model is given by

$$
\begin{aligned}
Z_{N}\left(f_{N}\right) & =\int \prod_{i=1}^{N} \mathrm{~d} \phi_{i} \sin \theta_{i} \mathrm{~d} \theta_{i} \exp \left(\frac{f_{N}}{k_{B} T} \sum_{i=1}^{N} b_{i} \cos \theta_{i}\right) \\
& =(4 \pi)^{N} \prod_{i=1}^{N}\left(\frac{k_{B} T}{b_{i} f_{N}}\right) \cosh \left(\frac{b_{i} f_{N}}{k_{B} T}\right) .
\end{aligned}
$$

The average orientation of the $i$-th bond vector can be calculated as

$$
\begin{aligned}
\left\langle\cos \theta_{i}\right\rangle & =\left(\frac{k_{B} T}{f_{N}}\right) \frac{\partial}{\partial b_{i}} \ln Z_{N}\left(f_{N}\right) \\
& =\operatorname{coth}\left(\frac{b_{i} f_{N}}{k_{B} T}\right)-\left(\frac{k_{B} T}{b_{i} f_{N}}\right) .
\end{aligned}
$$

From eq. (A.3) the chain-end mean distance $h$ is given by

$$
h=\sum_{i=1}^{N} b_{i}\left\langle\cos \theta_{i}\right\rangle=b N \mathcal{L}\left(\frac{b f_{N}}{k_{B} T}\right),
$$

where we have taken into account that the lengths of all bond vectors are the same, $b_{i}=b$, and where $\mathcal{L}(x)=$ $\operatorname{coth}(x)-1 / x$ is the Langevin function. This leads to the force-distance relationship

$$
\frac{b f_{N}}{k_{B} T}=\mathcal{L}^{-1}\left(\frac{h}{b N}\right)=\left\{\begin{array}{l}
\frac{1}{1-h / b N}, \quad \text { at } h / b N \leq 1, \\
\frac{2 h}{b N}, \quad \text { at } h / b N \ll 1,
\end{array}\right.
$$

which we use in sect. 2 . The notation $\mathcal{L}^{-1}(x)$ stands for the inverse Langevin function.

\section{Appendix B. Calculation of PDF in the h-ensemble}

Using eq. (24) for $\ln z^{*}(w)$ as well as eqs. (12) and (13) (for the BS model), one obtains an expression for the tail length

$$
M(h, \xi w)=\frac{h / l_{0}}{\left[a_{1}^{\phi}\left(w-w_{c}\right) \xi\right]^{(1-\nu) / \phi}} .
$$

The saddle point (SP) equation in this case reads (cf. eq. (21))

$$
\begin{aligned}
\frac{K+1}{\xi_{0}}= & -\left.\left[N-M\left(h, \xi_{0} w\right)\right] \frac{\partial \ln z^{*}}{\partial \xi}\right|_{\xi=\xi_{0}}+\left.\frac{\partial M}{\partial \xi}\right|_{\xi=\xi_{0}} \\
& \times \ln z^{*}\left(\xi_{0} w\right) .
\end{aligned}
$$

Taking into account eqs. (24) and (B.1), after introducing the notation $y=\left(w-w_{c}\right) \xi_{0}$, the $\mathrm{SP}$ equation can be recast in the form

$$
\left(\frac{K}{N}\right)=y^{1 / \phi}-B_{1}\left(\frac{h}{l_{0} N}\right) y^{\nu / \phi}-B_{2}\left(\frac{h}{l_{0} N}\right) \frac{\ln \mu_{3}}{y^{(1-\nu) / \phi}},
$$

where $B_{1}$ and $B_{2}$ are constants of the order of unity. In the particular case $h=0$, eq. (B.3) goes back, as expected, to eq. (26). Providing that $h \ll l_{0} N$, eq. (B.3) can be solved iteratively as

$$
\begin{aligned}
y=\left[\left(\frac{K}{N}\right)+B_{1}\left(\frac{K}{N}\right)^{\nu}\left(\frac{h}{l_{0} N}\right)\right. \\
\left.+B_{2} \ln \mu_{3}\left(\frac{N}{K}\right)^{1-\nu}\left(\frac{h}{l_{0} N}\right)\right]^{\phi} .
\end{aligned}
$$

As before the main contribution in the integral given by eq. (47) reads

$$
\begin{aligned}
& P(K) \propto \exp \left\{-\left[N-M\left(h, \xi_{0} w\right)\right] \ln z^{*}\left(\xi_{0} w\right)\right. \\
& \left.-(K+1) \ln \xi_{0}\right\}=\left\{\left(w-w_{c}\right) N^{\phi}\right. \\
& \times \exp \left[1-\frac{\left(h / l_{0} N\right)}{(K / N)^{1-\nu}\left[\rho\left(K / N, h / l_{0} N\right)\right]^{1-\nu}}\right] \\
& \left.\times \rho\left(K / N, h / l_{0} N\right)\right\}^{K}\left[K \rho\left(K / N, h / l_{0} N\right)\right]^{-\phi K},
\end{aligned}
$$

where one introduces the notation

$\rho\left(K / N, h / l_{0} N\right) \equiv 1+B_{1} \frac{h / l_{0} N}{(K / N)^{1-\nu}}+B_{2} \ln \mu_{3} \frac{\left(h / l_{0} N\right)}{(K / N)^{2-\nu}}$.

After normalization, the final expression for the PDF reads

$$
\begin{aligned}
& P(K)=\frac{1}{\mathcal{W}\left(\eta, h / l_{0} N\right)} \\
& \times\left\{\eta \exp \left[1-\frac{\left(h / l_{0} N\right)}{(K / N)^{1-\nu}\left[\rho\left(K / N, h / l_{0} N\right)\right]^{1-\nu}}\right]\right. \\
& \left.\times \rho\left(K / N, h / l_{0} N\right)\right\}^{K}\left[K \rho\left(K / N, h / l_{0} N\right)\right]^{-\phi K},
\end{aligned}
$$

where, as before, $\eta=\left(w-w_{c}\right) N^{\phi}$ and the normalization constant reads

$$
\begin{aligned}
& \mathcal{W}\left(\eta, h / l_{0} N\right)= \\
& \sum_{K=1}^{N}\left\{\eta \exp \left[1-\frac{\left(h / l_{0} N\right)}{(K / N)^{1-\nu}\left[\rho\left(K / N, h / l_{0} N\right)\right]^{1-\nu}}\right]\right. \\
& \left.\times \rho\left(K / N, h / l_{0} N\right)\right\}^{K}\left[K \rho\left(K / N, h / l_{0} N\right)\right]^{-\phi K} .
\end{aligned}
$$

Again one can readily see that for $h=0$ eq. (B.7) reduces to eq. (28). The PDF, following from eq. (B.7) is shown in 
fig. $3 \mathrm{~b}$ for several values of the height $h$. Evidently, both the mean value $\bar{K}$ and the variance $\overline{K^{2}}-\bar{K}^{2}$ decline with growing $h$.

\section{References}

1. S.B. Smith, Y. Cui, C. Bustamante, Science 271, 795 (1996).

2. T.J. Senden, J.-M. di Meglio, P. Auroy, Eur. Phys. J. B 3, 211 (1998).

3. T. Hugel, M. Grosholz, H. Clausen-Schaumann, A. Pfau, H.E. Gaub, M. Seitz, Macromolecules 34, 1039 (2001).

4. H. Hugel, M. Seitz, Macromol. Rapid. Commun. 22, 989 (2001).

5. A. Rohrbach, E.H.K. Stelzer, J. Appl. Phys. 91, 5474 (2002).

6. L. Sonnenberg, Y. Luo, H. Schaad, M. Seitz, H. Gölfen, H.E. Gaub, J. Am. Chem. Soc. 129, 15364 (2007).

7. C. Friedsam, A. Del Campo Becares, M. Seitz, H.E. Gaub, New J. Phys. 6, 9 (2004).

8. S.K. Kufer, E.M. Puchner, H. Gumpp, T. Liedl, H.E. Gaub, Science 319, 594 (2008).

9. M. Rief, F. Oesterhelt, B. Heymann, H.E. Gaub, Science 275, 1295 (1997).

10. C. Ortiz, G. Hadziioannou, Macromolecules 32, 780 (1990).

11. M. Grandbois, M. Beyer, M. Rief, H. Clausen-Schaumann, H.E. Gaub, Science 283, 1727 (1999).
12. A.M. Skvortsov, L.I. Klushin, T.M. Birshtein, Polymer Sci. (Moscow) Ser. A 51, 1 (2009).

13. S. Bhattacharya, V.G. Rostiashvili, A. Milchev, T.A. Vilgis, Phys. Rev. E 79, 030802(R) (2009).

14. S. Bhattacharya, V.G. Rostiashvili, A. Milchev, T.A. Vilgis, Macromolecules 42, 2236 (2009).

15. C. Vanderzande, Lattice Model of Polymers (Cambridge University Press, Cambridge, 1998).

16. E. Eisenriegler, K. Kremer, K. Binder, J. Chem. Phys. 77, 6296 (1982).

17. E. Eisenriegler, Polymers Near Surfaces (World Scientific, 1993).

18. T. Kreer, S. Metzger, M. Müller, K. Binder, J. Chem. Phys. 120, 4012 (2004).

19. A.Yu. Grosberg, A.R. Khokhlov, Statistical Physics of Macromolecules (AIP, New York, 1994).

20. J. des Cloiseaus, G. Jannink, Polymers in Solution (Clarendon Press, Oxford, 1990).

21. P.-G. de Gennes, Scaling Concept in Polymer Physics (Cornell University Press, 1979).

22. Y.-J. Sheng, P.-Y. Lai, Phys. Rev. E 56, 1900 (1997).

23. M. Wittkop, J.-U. Sommer, S. Kreitmeier, D. Göritz, Phys. Rev. E 49, 5472 (1994).

24. J.U. Schurr, S.B. Smith, Biopolymers 29, 1161 (1990).

25. J. Rudnick, G. Gaspari, Elements of Random Walk (Cambridge University Press, Cambridge, 2004).

26. S. Bhattacharya, H.-P. Hsu, V.G. Rostiashvili, A. Milchev, T.A. Vilgis, Macromolecules 41, 2920 (2008).

27. K. Binder, A. Milchev, J. Computer-Aided Material Design 9, 33 (2002). 